\title{
Study of Ultrathin In-Ag Layers on Tungsten by Means of Surface Spectroscopy Techniques
}

\author{
A. BukaluK*, M. Trzcinski and K. OKulewicz
}

Institute of Mathematics and Physics

University of Technology and Life Sciences

Kaliskiego 7, PL-85-796 Bydgoszcz, Poland

Auger electron spectroscopy, X-ray photoelectron spectroscopy, ultraviolet photoelectron spectroscopy, scanning tunnelling microscopy, low energy electron diffraction, and thermodesorption methods were used to investigate the process of growth of ultrathin In films and formation of $\mathrm{In}-\mathrm{Ag}$ surface alloys on tungsten substrate. Several indium layers, having coverage ranging from 0.2 to $0.8 \mathrm{ML}$ were deposited on tungsten substrate in room temperature. It was found that at the lowest coverages $(\Theta=0.1-0.3 \mathrm{ML})$ indium atoms form $(3 \times 1)$ structure, characterized by very sharp low energy electron diffraction patterns. With increasing $\Theta$ they tend to form densely packed islands interpreted as slightly distorted In(111) monolayers. Surface diffusion of In onto the tungsten surface was studied by using ESCA imaging property of SCIENTA ESCA200 instrument. Measuring the photoelectron intensity as a function of two spatial coordinates and the energy, we observed movement of In atoms on the tungsten surface. On the basis of the change of surface coverage with distance surface the diffusion coefficient was evaluated at the temperature range of 400-700 K. Intermixing of ultrathin films of indium and silver, after their surface diffusion from the sample edges to the centre of the tungsten surface, were observed by using X-ray photoelectron spectroscopy, ultraviolet photoelectron spectroscopy and the Auger electron spectroscopy methods. Intermixing was controlled by means of photoemission spectra from the valence states and the In $4 d$ level, as well as by X-ray photoelectron spectroscopy studies of indium and silver core levels excited by $\mathrm{Al} K_{\alpha}$ source.

PACS numbers: 61.05.jh, 61.46.Hk, 61.66.Dk, 68.37.Ef

\section{Introduction}

Interest in the processes related to the intermixing of ultrathin layers and further formation of surface alloys has intensified significantly in recent years, and new materials originating as a result of such surface material technology have al-

*corresponding author; e-mail: bukaluk@utp.edu.pl 
ready appeared in range of commercial applications. In spite of this rapid advance, the precise relationship between mechanisms of growth of ultrathin metal layers and formation of surface alloys is still not fully understood and there is a need for detailed studies of such systems.

Over the last years ultrathin metallic films grown on metal surfaces have been the subject of several studies because they can have very unusual properties: their surface chemistry as well as their electronic properties can be very different from those of their bulk $[1,2]$. These differences can be associated with both the interfacial interactions with the substrate and the spatial confinement and often they cause important differences in the crystal structure compared to the case when the same material adopts in the bulk phase.

One of the materials, recently extensively studied, is $\mathrm{In}-\mathrm{Ag}$ alloy. Silver is an element with a filled valence $d$-shell and isostructural (fcc) material in the bulk. High electrical and thermal conductivity of Ag makes the most of these materials a good component in electrical contacts. Indium is a $s p$ element with $s^{2} p^{1}$ outer valence shell configuration. It crystallizes in a close packed face-centred-tetragonal (fct) structure. Since indium is extremely malleable with excellent fatigue resistance, it is used as good lubricant, material for protective coatings against corrosion and for bonding delicate devices that need a low process temperature. Therefore, indium solder can be considered as bonding material between substrate and semiconductor chip in laser diodes, optoelectronic devices and photonic switches [3]. In order to ensure proper electrical, mechanical and antidiffusive properties of microelectronic devices, indium-based alloys are formed.

Nanostructured materials potentially create novel physical properties of metallic multilayers influenced by grain size, texture and interface morphology including interaction between constituents involved in interdiffusion. The latter leads to the diffusion soldering which can form strong and high-temperature stable bonds during relatively short bonding times at a moderate temperature. It occurs if the formation of intermetallic compounds or alloys is the dominating process in the joints [4]. It was found that the reaction proceeds if one metal has high melting point and the other one has low melting point [5]. In-Ag alloys seem to be good candidates for this purpose.

When indium film is evaporated onto the silver layer, Ag atoms can diffuse into In and form a number of intermetallic compounds. The interdiffusion in thin film Ag/In couples was studied by Simič and Marinkovič [6], Roy and Sen [7], and Lin et al. [8]. It was found that mass transport in thin film $\mathrm{Ag} / \mathrm{In}$ system occurs mainly via interstitial diffusion of $\mathrm{Ag}$ in In and grain boundary diffusion of In in $\mathrm{Ag}$ [7]. It was also indicated for samples having less than $55 \mathrm{wt} . \%$ In that both $\mathrm{Ag}_{2} \mathrm{In}$ and $\mathrm{AgIn}_{2}$ phases are formed. Therefore, for the potential applications of the In-Ag films detailed studies of their properties are necessary to be performed.

Besides of the application possibilities of In-Ag surface alloys, studies of these systems are also interesting from the pure cognitive reasons. In our pre- 
vious papers we have studied mechanism of growth of In ultrathin layers on the $\mathrm{W}(110)$ by means of photoelectron spectroscopy combined with low energy electron diffraction (LEED) [9], and by scanning tunnelling microscopy (STM) and LEED methods [10]. Preliminary studies of In diffusion on W(110) surface [11] as well as intermixing in the $\mathrm{In} / \mathrm{Ag} / \mathrm{W}(110)$ and $\mathrm{Ag} / \mathrm{In} / \mathrm{W}(110)$ structures [12] have also been performed. Therefore we have found it interesting to complete studies of ultrathin In layers formation on the tungsten surface by using the Auger electron spectroscopy (AES)/LEED and thermodesorption spectroscopy (TDS) experimental methods. Some additional surface diffusion studies of In on $\mathrm{W}$ and $\mathrm{Ag}$ on $\mathrm{W}$ and further intermixing have been planned.

Indium is an element distinctly different electronically than silver. It has a $s^{2} p^{1}$ outer valence shell configuration and the closed $4 d^{10}$ shell is observed around $17 \mathrm{eV}$ below the energy of the Fermi level, so it is well separated from the $4 d^{10}$ bands of silver. The binding energy of In $4 d$ is too high to be excited by $\mathrm{He}(\mathrm{II})$ radiation $(\hbar \omega=40.8 \mathrm{eV})$ simultaneously with $\mathrm{Ag} 4 d$, and at much better electron energy resolution compared to X-ray induced core-level photoemission.

Studies of surface properties of thin films of indium and silver are not numerous [6-8], and most of them concern investigations of properties of films with thickness of hundreds of monolayers. In our previous studies of intermixing in the submonolayer range of $\mathrm{In}-\mathrm{Ag}$ structures we proved formation of surface alloys in the whole range of In and $\mathrm{Ag}$ coverages for the structures $\mathrm{In} / \mathrm{Ag} / \mathrm{W}$ and $\mathrm{Ag} / \mathrm{In} / \mathrm{W}$ [12]. Nowadays we found it interesting to investigate intermixing of ultrathin $\mathrm{In}-\mathrm{Ag}$ layers once again, observing surface alloying of In and Ag after diffusion of both elements from the opposite edges of the tungsten substrate.

We have performed our investigations on the tungsten substrate, since it is experimentally convenient for the epitaxial growth of several metals and metal alloys. In particular with noble metal overlayers no alloying occurs and the sample can be cleaned after each deposition step just by thermal desorption of the adsorbate [13]. No dissolution and alloying of indium in tungsten and formation of intermetallic compounds were also found for the In-W system [14].

\section{Experimental}

We have performed electron spectroscopy studies by means of SCIENTA photoelectron spectrometer. X-ray photoelectron spectra (XPS) spectra have been obtained by using monochromatised $\mathrm{Al} K_{\alpha}$ radiation source $(\hbar \omega=1486.6 \mathrm{eV})$, with energy resolution $\Delta E=100 \mathrm{meV}$. Ultraviolet photoelectron spectra (UPS) spectra have been taken by means of monochromatised resonance radiation from $\mathrm{He}(\mathrm{I})(\hbar \omega=21.2 \mathrm{eV})$, and $\mathrm{He}(\mathrm{II})(\hbar \omega=40.8 \mathrm{eV})$, with energy resolution $\Delta E=$ $10 \mathrm{meV}$. LEED and AES studies have been performed by using home-made Video-LEED system based on VG camera. All experiments were performed in the UHV chamber with a base pressure of $1 \times 10^{-10}$ mbar.

In investigations of the process of ultrathin In layers formation on the tungsten substrate we employed a W(110) crystal oriented with an accuracy of $\pm 0.25^{\circ}$. 
It was $1.5 \mathrm{~mm}$ thick round plate with $10 \mathrm{~mm}$ diameter. Its surface was cleaned by annealing in $5 \times 10^{-8}$ mbar of oxygen at $1400 \mathrm{~K}$, followed by a flash to $2300 \mathrm{~K}$. Surface cleanliness was controlled by the absence of contamination lines in core-level photoelectron spectra, checked by XPS and UPS. Surface diffusion studies were performed on polycrystalline tungsten substrate of $99.999 \%$ purity. It was $2 \mathrm{~mm}$ thick and with $12 \mathrm{~mm}$ diameter. Surface of polycrystalline substrate was prepared in the same way as $\mathrm{W}(110)$ crystal before indium and silver evaporation on the opposite edges of the tungsten substrate.

Indium was evaporated from PBN crucible. During deposition the substrate was held at room temperature. Deposition rate of In was approximately $0.2 \mathrm{ML} / \mathrm{min}$, where $1 \mathrm{ML}$ corresponds to the atomic density in the top most layer of an ideal W(110) surface. To monitor the amount of In we have used the XPS intensities of $3 d$ and $4 d$ core levels of In and the $4 p_{3 / 2}$ and $4 f_{7 / 2}$ levels of $\mathrm{W}$. On the basis of the intensity ratio $R$ of $\mathrm{In} 3 d_{5 / 2}$ and $\mathrm{W} 4 p_{3 / 2}$ intensities calculated from the area below the peaks we obtained the overlayer coverage from the formula: $\Theta=\lambda R \sqrt{2} \sigma(\mathrm{W}) /[\sigma(\mathrm{In}) a]$, where $\lambda$ is the electron mean free path, $R=I\left(\operatorname{In} 3 d_{5 / 2}\right) / I\left(\mathrm{~W} 4 p_{3 / 2}\right), \sigma(W) / \sigma(\operatorname{In})$ is the cross-section ratio and $a$ is the tungsten bulk lattice constant [9].

Silver was also evaporated from the PBN crucible at the same rate as indium, i.e. about $0.2 \mathrm{ML}$ per minute. Silver coverages were determined by using the XPS intensities of the $\mathrm{Ag} 3 d$ and $\mathrm{W} 4 d$ core levels. The absolute calibration was performed by combination of LEED and UPS results as well as on the Ag-induced change in work function $\Delta \Phi[13]$. All depositions were performed at room temperature of the substrate.

\section{LEED and AES studies of ultrathin In layers formation on the $W(110)$ surface}

We started LEED studies from the observation of the patterns originating from pure W(110) substrate. Figure 1a presents LEED pattern corresponding to pure tungsten $(110)$ surface. The substrate $(1 \times 1)$ LEED spot positions are shown by the large open circles. Figures $1 \mathrm{~b}-\mathrm{e}$ present evolution of LEED patterns after deposition of different amounts of In. Figure $1 \mathrm{~b}$ reproduces schematically the LEED pattern observed very clearly and at low background intensity after deposition of a layer with a nominal thickness of $0.2 \mathrm{ML}$. The black dots, corresponding to positions of In atoms, clearly demonstrate that In is ordered, with an In-In distance exceeding the $\mathrm{W}-\mathrm{W}$ distance. In our previous LEED and STM investigations we proved that the pattern corresponds to the $(3 \times 1)$ superstructure [10].

At the overlayer thickness of $0.45 \mathrm{ML}$ we observed that additional pattern, shown as grey dots, is superimposed to that shown in Fig. 1b. Further In deposition of additional 0.25 ML resulted in disappearance of the pattern shown in Fig. 1b and the superimposed pattern from Fig. 1c became more distinct. Comparative STM and LEED studies [10] allowed to indicate that $(1 \times 4)$ superstructure started 
(a)

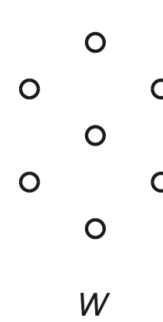

(b)

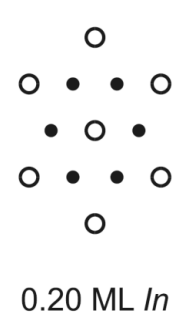

(c)

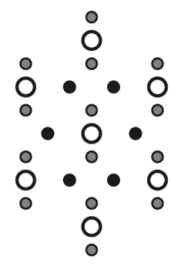

$0.45 \mathrm{ML} / n$ (d)

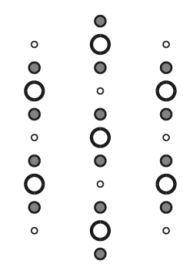

$0.65 \mathrm{ML} / n$ (e)

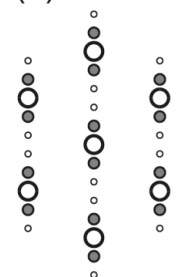

$0.80 \mathrm{ML} / n$

Fig. 1. LEED patterns corresponding to the different amounts of In deposited on tungsten: (a) pure W(110), (b) 0.2 ML In on W(110), (c) $0.45 \mathrm{ML}$ In on W(110), (d) 0.65 ML In on W(110), (e) 0.80 ML In on W(110).

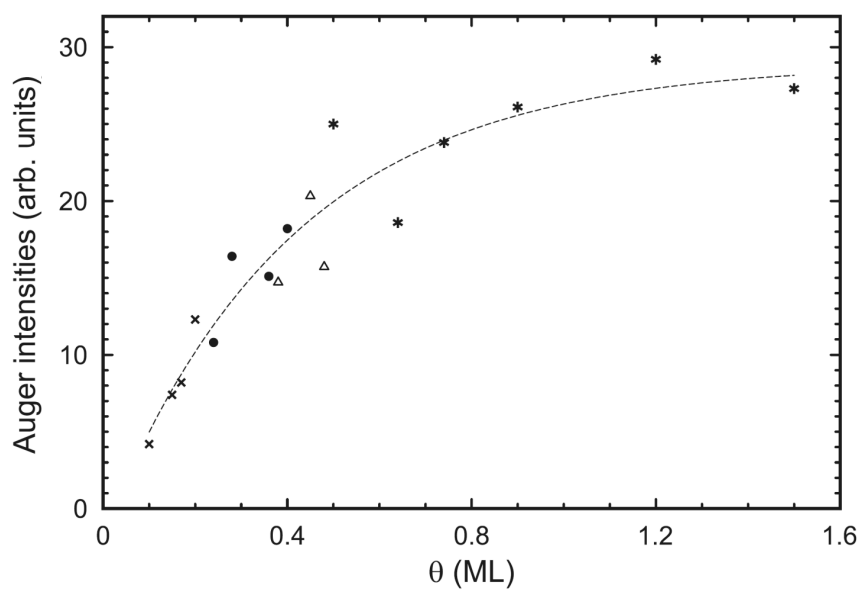

Fig. 2. Dependence of Auger intensities versus In coverage corresponding to the following LEED patterns: $\times-0.20 \mathrm{ML}$ In on W(110) (Fig. 1b), • - $0.45 \mathrm{ML}$ In on W(110) (Fig. 1c), $\Delta-0.65$ ML In on W(110) (Fig. 1d), *-0.80 ML In on W(110) (Fig. 1e).

to be formed on the W(110) surface in that case. Further deposition of indium resulted in the LEED pattern shown in Fig. 1d. Deposition of additional 0.2 ML resulted in transformation of $(1 \times 4)$ superstructure into the $(1 \times 5)$ structure, shown in Fig. 1e, observed at coverages exceeding $0.8 \mathrm{ML}$.

It was also noticed that while $(1 \times 4)$ and $(1 \times 5)$ structures remained stable, the $(3 \times 1)$ superstructure appeared to be unstable and it was found that it transformed into a $(1 \times 4)$ pattern within $1-2 \mathrm{~h}$ after deposition at room temperature. This transformation also occurred almost immediately after annealing.

From these observations we conclude that $(3 \times 1)$ structure is only metastable, whereas the $(1 \times 4)$ and $(1 \times 5)$ overlayers are thermodynamically stable phases. In the $(3 \times 1)$ structure the indium atoms are only loosely packed together. STM studies indicated that upon aging or annealing the atoms apparently agglomerate 
into islands of higher local coverage. Locally, these islands have the same structure as the stable global $(1 \times 4)$ phase usually observed at higher coverages.

Figure 2 presents Auger peak-to-peak heights (APPH's) vs. In coverage registered for $(3 \times 1),(1 \times 4)$ and $(1 \times 5)$ superstructures. One can see that APPH's reveal rapid increase in the range from 0.2 to about $0.45 \mathrm{ML}$, and then the Auger intensity slowly saturates. This dependence can be also used for scaling of indium coverages on the basis of the Auger measurements. Consecutive STM studies of indium overlayers formed on the W(110) surface indicate that $(1 \times 4)$ and $(1 \times 5)$ superstructures observed close to completion of the first monolayer can be interpreted as slightly distorted $\operatorname{In}(111)$ monolayers in the NishiyamaWassermann orientation. The indium density in the $(1 \times 4)$ superstructure almost perfectly matches that of $\operatorname{In}(111)$ monolayer, rendering this structure particularly stable.

\section{Surface diffusion of In atoms on the polycrystalline tungsten surface}

We started investigations of migration of In atoms on the polycrystalline tungsten surface from the thermodesorption measurements. After evaporation of In on the crystal surface we rapidly heated the tungsten substrate (usually $2 \mathrm{~s}$ ) to the desired temperature, held it for $30 \mathrm{~s}$ in a given temperature, cooled the crystal and then performed the XPS measurement. Then the whole procedure was repeated, consecutively increasing temperature in each stage, until the surface was clean. Results of TDS measurements have been shown in Fig. 3. From this figure one can state that thermodesorption of the film with thickness of $6-2$ monolayers occurs in the narrow temperature range 700-800 K. For this part of thermodesorption curve we can apply the zero-order desorption process [15], described by the formula

$$
-\mathrm{d} \Theta / \mathrm{d} t=\nu \exp \left(-E_{\mathrm{d}} / R T\right),
$$

where $\nu$ is frequency factor and $E_{\mathrm{d}}$ is the activation energy of desorption. On the basis of this formula we evaluated $E_{\mathrm{d}}=225 \mathrm{~kJ} \mathrm{~mol}^{-1}$, which is in reasonable agreement with the sublimation energy of In $\left(215 \mathrm{~kJ} \mathrm{~mol}^{-1}\right)$. Thermodesorption curve for the temperatures higher than $800 \mathrm{~K}$ is more complex. From $810 \mathrm{~K}$ to $910 \mathrm{~K}$ desorption of the second monolayer occurs. At the temperature of $810 \mathrm{~K}$ for the coverage of about 2.0 ML we notice a break in TDS plot. Similar "knee" is observed at $1020 \mathrm{~K}$ for the coverage about 1.0 ML. These distinct "knees" on the TDS spectra suggest that In starts to grow on the polycrystalline tungsten surface in the layer-by-layer manner. This agrees well with the earlier work of Ciszewski [16], who observed that thick In films (10-100 layers) grow epitaxially on tungsten surface. These observations performed on a highly-stepped and curved surface used in a field-emission microscope are not directly comparable to our results. However, they indicate that epitaxial relationship between In and W can be expected. 


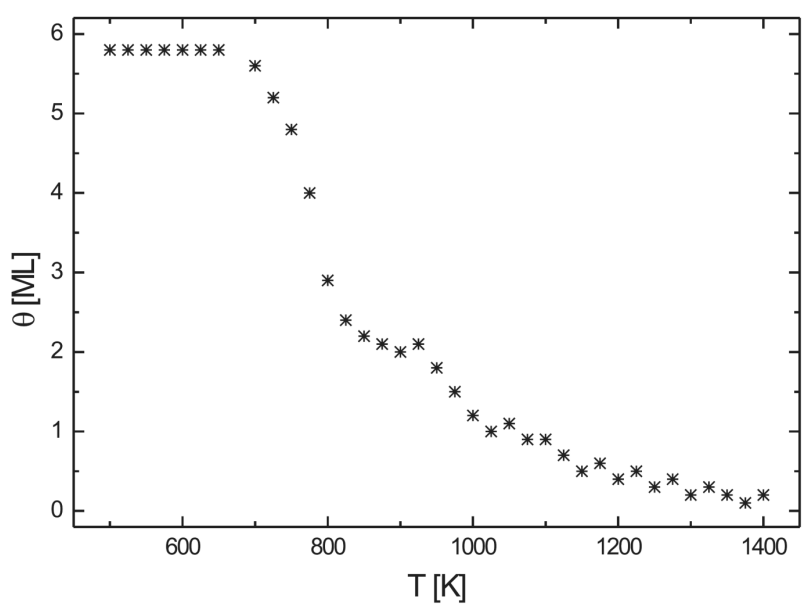

Fig. 3. Thermodesorption spectrum of In layer of initial thickness 5.8 ML.

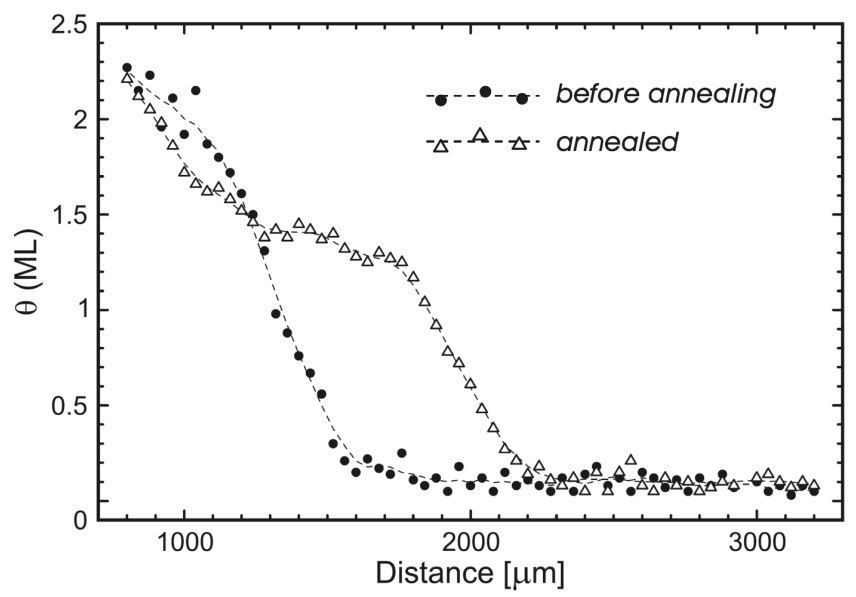

Fig. 4. Diffusion profile of In on polycrystalline W surface: $\Theta(x)$ dependence for In layer of preliminary thickness $2.3 \mathrm{ML}$, annealed for 5 min at $680 \mathrm{~K}$, in comparison with the unheated sample.

To get more information about the behaviour of indium on tungsten we employed imaging property of SCIENTA instrument [17]. Indium films of 2.0-2.8 ML thickness have been deposited on the area a little smaller than one half of the tungsten surface. Then the sample has been annealed to the temperatures 400-700 K and In transportation as well as its further formation on the surface was observed. Measuring the photoelectron intensity of In $3 d_{5 / 2}$ signal as a function of distance and energy we monitored the process of In diffusion on the surface. Then, on the basis of the above-mentioned scaling procedure we transformed In $3 d_{5 / 2}$ signal intensities into indium coverage $\Theta$. Figure 4 shows $\Theta(x)$ dependence 
TABLE

Values of surface diffusion coefficients of indium on the polycrystalline tungsten substrate.

\begin{tabular}{c|c}
\hline \hline Temperature $[\mathrm{K}]$ & Diffusion coefficient $\left[\mathrm{m}^{2} \mathrm{~s}^{-1}\right]$ \\
\hline 421 & $8.9 \times 10^{-11}$ \\
460 & $1.6 \times 10^{-10}$ \\
517 & $2.0 \times 10^{-10}$ \\
564 & $2.3 \times 10^{-10}$ \\
615 & $2.6 \times 10^{-10}$ \\
645 & $3.0 \times 10^{-10}$ \\
680 & $4.5 \times 10^{-10}$
\end{tabular}

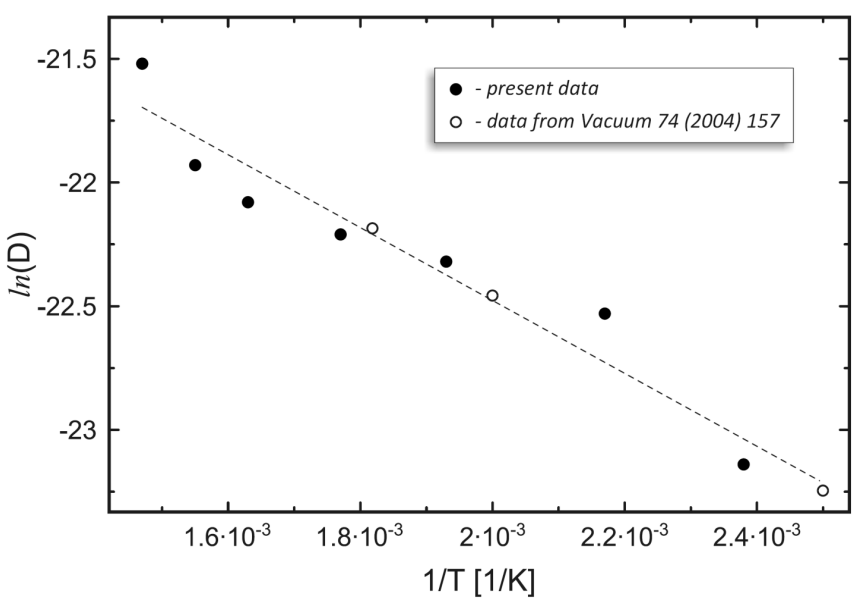

Fig. 5. Arrhenius plot of the surface diffusion coefficients: $\bullet-$ present data, $\circ-$ data obtained previously for In diffusion on W(110) [11].

for the sample heated for $5 \mathrm{~min}$ at $680 \mathrm{~K}$. One can see that annealing caused movement of the edge of In layer over the distance of about $500 \mu \mathrm{m}$. Diffusion data were analysed using the expression allowing evaluation of the surface diffusion coefficient from the shape of diffusion plots [14]:

$$
\int_{a}^{b}\left(\Theta-\Theta_{0}\right) \mathrm{d} x=D t\left[\left(\frac{\mathrm{d} \Theta}{\mathrm{d} x}\right)_{a}-\left(\frac{\mathrm{d} \Theta}{\mathrm{d} x}\right)_{b}\right],
$$

where $a$ and $b$ are chosen values of the distance $x$ across the profile, $\Theta_{0}$ and $\Theta$ are the values of the coverage for a given $x$ and for the profile of the unheated sample and annealed one, respectively, $t$ is the time of annealing and $D$ is the so-called chemical surface diffusion coefficient.

In the similar way we have found $\Theta(x)$ dependences for six other temperatures from the range $421-680 \mathrm{~K}$. The values of surface diffusion coefficients 
have been evaluated from the dependence (2) and have been presented in Table. They are generally consistent with the values obtained for indium diffusion on tungsten surface at coverages $\Theta=1-2 \mathrm{ML}$, extrapolated to the temperatures 400-700 K [14]. They are also consistent with the data obtained for surface diffusion of other adsorbates - Li, Ba and $\mathrm{O}$ on tungsten [18-20]. Figure 5 shows surface diffusion data presented in the form of the Arrhenius plot. Although in most cases surface diffusion coefficients are not only coverage dependent, but also temperature dependent $[14,19]$, in our case one can see that experimental data fit the linear dependence in the Arrhenius plot quite well. Moreover, three experimental points obtained previously for In surface diffusion on W(110) fit the line in reasonable manner. The values of the surface diffusion pre-exponential factor $D_{0}=3.1 \times 10^{-9} \mathrm{~m}^{2} \mathrm{~s}^{-1}$ and the activation energy $E_{\mathrm{a}}=12.02 \mathrm{~kJ} / \mathrm{mol}$ have been found from the linear dependence plotted in Fig. 5.

\section{Formation of $A g_{x} \operatorname{In}_{1-x}$ surface alloy after $\mathrm{In}$ and $\mathrm{Ag}$ surface diffusion on tungsten}

In the consecutive step of our studies we have deposited indium on the less than one half area of the polycrystalline $\mathrm{W}$ substrate, and the same amount of silver on the opposite edge of the tungsten surface. Then we heated the sample and observed the area in the centre of the substrate. In our studies we registered UPS spectra of $\mathrm{Ag} 4 d$ and In $4 d$ lines by using $\mathrm{He}(\mathrm{I})$ and $\mathrm{He}(\mathrm{II})$ monochromatised radiation. The $\mathrm{Ag} 4 d$ spectrum revealed two most prominent lines observed at $4.55 \pm 0.05 \mathrm{eV}$ and $4.93 \pm 0.05 \mathrm{eV}$, corresponding to photoemission from the $\mathrm{Ag} 4 d$ valence orbitals, in good agreement with the results obtained earlier for the $\mathrm{Ag} / \mathrm{W}(110)$ system $[9,21-23]$. In the In $4 d$ spectrum one could notice two distinct lines corresponding to $4 d_{5 / 2}$ and $4 d_{3 / 2}$ transitions in indium. Spin-orbit splitting of about $0.9 \mathrm{eV}$ was in reasonable agreement with other studies [24, 25] performed for bulk alloys. Binding energy of the $4 d_{5 / 2}$ line was compatible with our previous XPS studies of In growth on the W(110) [9].

In description of the resulting surface structure we used convenient notation, introduced in our previous paper [9]: $\mathrm{Ag}_{x} \mathrm{In}_{1-x}$ denoted $\mathrm{In}-\mathrm{Ag}$ structure, in which $x$ indicated the relative amount of $\mathrm{Ag}$ present on the surface, whereas the total coverage $(\mathrm{Ag}+\mathrm{In})$ was given in brackets. It is necessary to indicate that this notation does not contain information neither on the overlayer geometry nor the degree of possible alloying.

Diffusion of In on $\mathrm{Ag}$ and then their intermixing in the centre of the tungsten substrate resulted in drastic change of the shape of the lines and the change of the binding energies $E_{\mathrm{b}}$. In Fig. 6 values of binding energy of In $4 d_{5 / 2}$ line have been marked by triangles and plotted as a function of In content in the $\mathrm{Ag}_{x} \mathrm{In}_{1-x}$ alloy. The values obtained for pure In layer have been marked by open circles, those obtained in [12] have been shown as crosses and present data have been marked by triangles. One can notice that the data related for higher coverages 


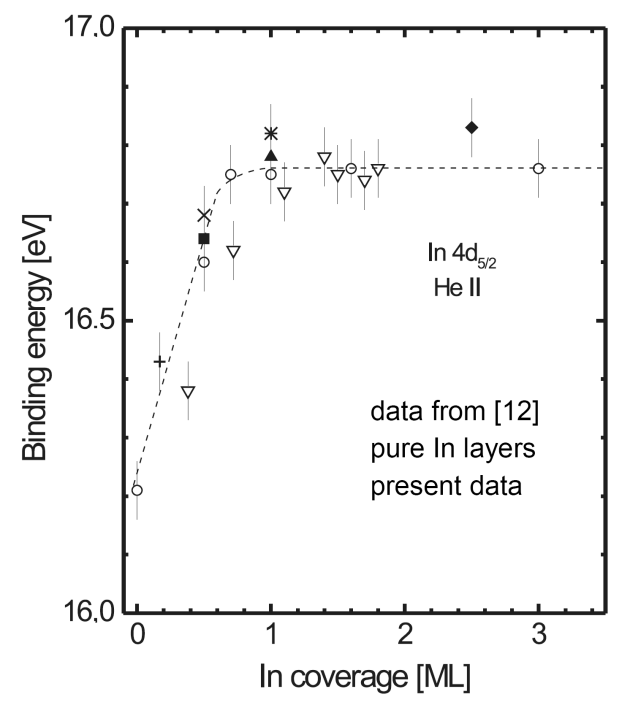

Fig. 6. In $4 d_{5 / 2}$ core level binding energies obtained for various $\operatorname{Ag}_{x} \operatorname{In}_{1-x}$ alloys in their dependence on In coverage.

are independent of composition and do not show significant binding energy shift. On the other hand, alloy data for In coverages fit very well to $E_{\mathrm{b}}(\Theta)$ dependence observed for pure In. Therefore one can state that the observed energy shift and the corresponding charge transfer are related to In-W interaction. Comparison of results obtained for bulk [26] and surface alloys indicate that they are very consistent.

Data presented in Fig. 6 show that binding energy of In $4 d_{5 / 2}$ line increases after intermixing with silver. However, the changes in binding energies are distinct for low coverages and small for In coverages exceeding 1 ML. On the other hand, for $\Theta>1$ data obtained in this study agree well with the binding energies of In $4 d$ in bulk alloys [25]. We have also noticed that after annealing the width of In $4 d$ doublet decreases by about $20 \%$.

All the observed features, like drastic energy shift and the change of the shape of $\mathrm{Ag} 4 d$ valence band, energy shift and narrowing of In $4 d$ line, confirm conclusions of previous studies [10] that effective intermixing of In and Ag occurs leading to the formation of surface alloy.

Dependence shown in Fig. 6 for lower In coverages show that $A g_{x} \operatorname{In}_{1-x}$ data fit the values obtained for pure In layer. Therefore, if the next In neighbour is replaced by Ag, the energy shift is almost unchanged. This confirms the fact that the binding energy shifts are related to In-W interaction and not to In-In, and is in good agreement with the mentioned-above mechanism of In growth. Silver diffusing onto the tungsten surface finds free places in the surface structures and forms $\mathrm{Ag}_{x} \operatorname{In}_{1-x}$ alloys. As a result, the change of line shape and binding energies of Ag $4 d$ line, and to less extent also of In $4 d$ lines occurs. 


\section{Conclusions}

Our studies confirmed previous suggestions that if In is deposited on $\mathrm{W}(110)$, it forms consecutively metastable $(3 \times 1),(1 \times 4)$ and at least $(1 \times 5)$ structures. Deposition of In onto the polycrystalline tungsten results in the layer-by-layer mode of growth. Intermixing of ultrathin indium and silver films deposited onto the opposite sides of the tungsten surface and then annealed, allowed to state that alloying process occurs. Evolution of In $4 d$ binding energy vs. In coverage indicates that the observed energy shift and the corresponding charge transfer are related to the In-W interaction, and are a consequence of the mechanism of In growth on the tungsten surface. Valence state data of $\mathrm{Ag} 4 d$ bands indicate complete intermixing, identical with alloying in $\mathrm{Ag}_{x} \operatorname{In}_{1-x}$ bulk alloys.

\section{Acknowledgments}

We are grateful to Prof. Erminald Bertel and Prof. Norbert Memmel from Leopold Franzens Universität Innsbruck, Prof. Albrecht Goldmann from Universität Kassel and to Prof. William Salaneck from Linköpings Universitet for their help in performing experimental work, and for many valuable suggestions and discussions. Financial support by the Polish Ministry of Science and Higher Education (project 1P03B 105 29) is also gratefully acknowledged.

\section{References}

[1] J.A. Rodriguez, Surf. Sci. Rep. 24, 223 (1996).

[2] H. Dreyssé, C. Demangeat, Surf. Sci. Rep. 28, 65 (1997).

[3] W.W. So, R. Chuang, C.C. Lee, Thin Solid Films 376, 164 (2000).

[4] T. Studnitzky, R. Schmid-Fetzer, Z. Metallkunde 93, 885 (2002).

[5] V. Simič, Ž. Marinkovič, Mater. Chem. Phys. 47, 246 (1997).

[6] V. Simič, Ž. Marinkovič, Thin Solid Films 61, 149 (1979).

[7] R. Roy, S.K. Sen, Thin Solid Films 197, 303 (1991).

[8] J.-C. Lin, L.-W. Hung, G.-Y. Jang, S.H. Lee, Thin Solid Films 410, 212 (2002).

[9] M. Bürgener, A. Bukaluk, M. Cyrankiewicz, A. Goldmann, R. Siuda, M. Trzcinski, Surf. Sci. 529, 490 (2003).

[10] M. Gabl, M. Trzcinski, N. Memmel, A. Bukaluk, E. Bertel, Surf. Sci. 600, 4390 (2006).

[11] M. Trzcinski, A. Bukaluk, A. Goldmann, M. Bürgener, Vacuum 74, 157 (2004).

[12] M. Trzcinski, A. Bukaluk, M. Bürgener, A. Goldmann, Surf. Sci. 589, 192 (2005).

[13] J. Feydt, A. Elbe, H. Engelhard, G. Meister, A. Goldmann, Surf. Sci. 452, 33 (2000).

[14] M.A. Morris, C.J. Barnes, D.A. King, Surf. Sci. 173, 618 (1986).

[15] D.A. King, T.E. Madey, J.T. Yates Jr., J. Chem. Phys. 55, 3236 (1971).

[16] A. Ciszewski, Surf. Sci. 83, 253 (1979). 
[17] U. Gelius, B. Wanneberg, P. Baltzer, H. Fellner-Feldegg, G. Carlsson, C.G. Johansson, J. Larsson, P. Münger, G. Vegerfors, J. Electron Spectrosc. Relat. Phenom. 52, 747 (1990).

[18] A.T. Laburets, A.G. Naumovets, Yu.S. Vedula, Surf. Sci. 120, 347 (1982).

[19] A.G. Naumovets, V.V. Poplavsky, Yu.S. Vedula, Surf. Sci. 200, 321 (1988).

[20] R. Butz, H. Wagner, Surf. Sci. 63, 448 (1977).

[21] E. Bauer, Appl. Surf. Sci. 11/12, 479 (1982).

[22] E. Bauer, H. Poppa, Thin Solid Films 121, 159 (1984).

[23] A. Elbe, G. Meister, A. Goldmann, Surf. Sci. 397, 346 (1998).

[24] S. Hüfner, Photoelectron Spectroscopy - Principles and Applications, Springer Series in Solid-State Science, Vol. 82, Springer, Berlin 1995.

[25] D.M. Riffe, G.K. Wertheim, D.N.E. Buchanan, P.H. Citrin, Phys. Rev. B 45, 6216 (1992).

[26] J.D. Riley, R.C.G. Leckey, J.G. Jenkin, J. Liesegang, R.T. Poole, J. Phys. F 6, 293 (1976). 